\title{
Color and Shape based Method for Detecting and Classifying Card Images
}

\author{
Cho Nilar Phyo ${ }^{1}$, Thi Thi Zin ${ }^{1}$, Hiroshi Kamada ${ }^{2}$, Takashi Toriu ${ }^{3}$ \\ ${ }^{1}$ Graduate School of Engineering, University of Miyazaki, Miyazaki, Japan \\ ${ }^{2}$ Graduate School of Engineering, Kanazawa Institute of Technology, Ishikawa, Japan \\ ${ }^{3}$ Graduate School of Engineering, Osaka City University, Osaka, Japan \\ nc16004@student.miyazaki-u.ac.jp, thithi@cc.miyazaki-u.ac.jp
}

\begin{abstract}
This paper proposes an effective method for detecting and classifying card images by using color and shape features We extract the card color area using color information and remove low possibility regions based on shape feature. Then, we classify the image by taking classroom size and camera distance. In order to confirm the proposed method, we conduct the experiments with our own videos. According to experimental results the proposed method achieves the overall accuracy of $93.93 \%$ in various classroom type (small and large).
\end{abstract}

Keywords: color segmentation, shape classification, interactive e-learning.

\section{Introduction}

In today world, the education system is rapidly changing and the video conferencing is widely used for education due to its enormous advantages. The video conferencing based e-learning system can make the classroom to be geographically limitless and reduce the travel cost and time for the lecturers. One weakness of the traditional video conferencing based e-learning system is the difficulty of getting student's feedback when it is using in enlarge classroom. The computer vision based interactive e-learning system can solve that weakness by supporting student-lecturer interaction during the lecture time which is the most important thing in teaching system. In the proposed methods, we use color and shape based color card detection and classification method for the implementation of interactive e-learning system. Five kinds of color cards are used to implement the system, so that the students can choose five kind of answers.

We describe some related work of shape based object identification and color based segmentation in section 2 and the detail process of the proposed method in section 3 . Then we describe some experimental results in section 4 and the conclusions of the proposed methods is explained in section 5 .

\section{Related Works}

This session describes about some interactive e-learning research work in the computer vision field. The authors proposed $^{1}$ the on-line assessment of user meta-cognitive behavior during the interaction with an intelligent learning environment by using eye-tracking data. The authors implement ${ }^{2}$ that interactive e-learning using head posture information such as students' face and hair information to identify a human presence and estimate the gaze direction. The e-learning system which consists of image recognition, color and polka-dot pattern recognition, and augmented reality engine with audiovisual contents is described in Ref. 3.

\section{Proposed System}

In this system, we will use color cards images to implement the interactive e-learning system in which the students can immediately give feedback over the understanding level of the lecture. So that the e-learning system can become more active and attractive and the lecturer can find the way to explain about the lecture based on the feedback of the students. There include four 
main components in the proposed system as shown in Fig. 1.

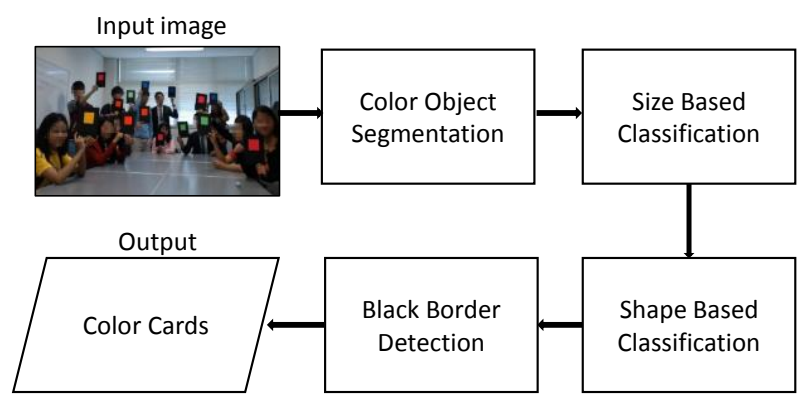

Fig. 1. Overview of the detection and classification of card images

\subsection{Color Object Segmentation}

The first component of the system is segmentation of color object. In the proposed system, we will use HSL color space in order to segment the color object under various illumination changing condition. In HSL color space, $\mathrm{H}$ stands for hue that measure the purity of the base color, $\mathrm{S}$ stands for saturation that measure the degree of white color embedded in specific color and L stands for lightness. HSL is the non-linear transformation of devicedependent RGB color model and widely used in the color picker systems of many graphical applications because of its convenience for the color perception of human being. We can simply transform HSL from RGB color space by performing the following steps:

(i) Find maximum(Max) and minimum (Min) value in $\mathrm{R}, \mathrm{G}$ and $\mathrm{B}$ component.

(ii) Find $\mathrm{L}$ value as describe the following.

$$
L=\frac{1}{2}(\operatorname{Max}+\operatorname{Min})
$$

(iii) Find $\mathrm{S}$ value using $\mathrm{L}$ value.

$$
S=\frac{\operatorname{Max}-\operatorname{Min}}{1-|2 * L-1|}
$$

(iv) Find $\mathrm{H}$ value using the following equation.

$$
H=\left\{\begin{array}{lll}
{\left[60 *\left(\frac{G-B}{\operatorname{Max}-\operatorname{Min}}\right)\right] \bmod 360} & \text { if } & \text { Max }=R \\
{\left[60 *\left(\frac{B-R}{\operatorname{Max}-\text { Min }}\right)\right]+120} & \text { if } & \text { Max }=G \\
{\left[60 *\left(\frac{R-G}{\operatorname{Max}-\text { Min }}\right)\right]+240} & \text { if } & \text { Max }=B
\end{array}\right.
$$

\subsubsection{Color Thresholding}

Thresholding is the important task in color segmentation and the performance of the system is depending on the choosing of correct color threshold. In our system, we use two threshold range for defining the card color (pink, yellow, green, blue and orange), one is for day time and another is for the night time. In order to determine the suitable threshold range, we vertically divide the image into two parts and check day time or night time by using the mean intensity of the upper part. If the mean intensity is greater than some threshold, we regard as day time image because the mean intensity of day time image is greater than night time. The result color mask of the segmentation of blue color region is as shown in Fig.2.

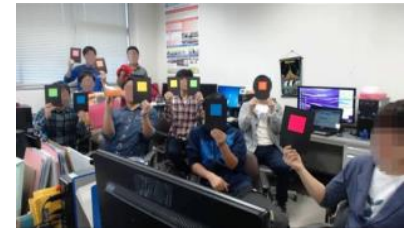

(a)

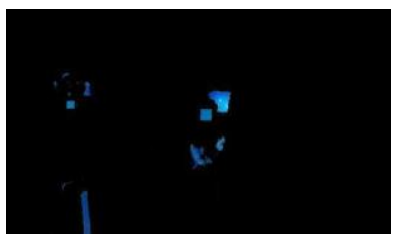

(b)
Fig. 2. (a) Input image, (b) Segmentation result of blue color

\subsection{Size Based Classification}

The second part of the system is classification of cards object using size information which is taking according to the classroom size. We use two threshold range in order to accuracy detect the card object in both far and near from the camera. To define far and near area, we vertically divide the image into two parts and regards the upper part as far area and the lower part as near area from the camera. Then we generate the equation for finding the size threshold depend on the relationship between 3D and $2 \mathrm{D}$ in photograph.

\subsubsection{Size Thresholding}

In order to find the size threshold, we take the photograph of the card by placing it on 1 to 5 meters' distance from the camera. Then, we count the height pixels of color region as shown in Table 1 . Then we generate the equation to estimate the height using the following linear equation.

$y=m * x+b$ 
where $\mathrm{y}$ is the height in pixel, $\mathrm{x}$ is the value $1 / \mathrm{D}, \mathrm{D}$ is the 3D real world distance between the card and camera, $m$ determines the slope of the straight line and $b$ is the interception point of $y$ axis. We get the value of 68 and 3 for parameter $m$ and $b$. distribution of the height and the straight line equation is shown in Fig. 3. The result color mask is as shown in Fig. 4.

Table 1 Estimated height of color region

\begin{tabular}{|c|c|c|c|c|c|}
\hline Distance (meter) & 1 & 2 & 3 & 4 & 5 \\
\hline 1/Distance & 1 & 0.5 & 0.33 & 0.25 & 0.2 \\
\hline height (pixel) & 71 & 37 & 26 & 20.0 & 17 \\
\hline Estimated height & 71 & 37 & 25.67 & 20 & 16.6 \\
\hline Error & 0 & 0 & 0.33 & 0 & 0.4 \\
\hline
\end{tabular}

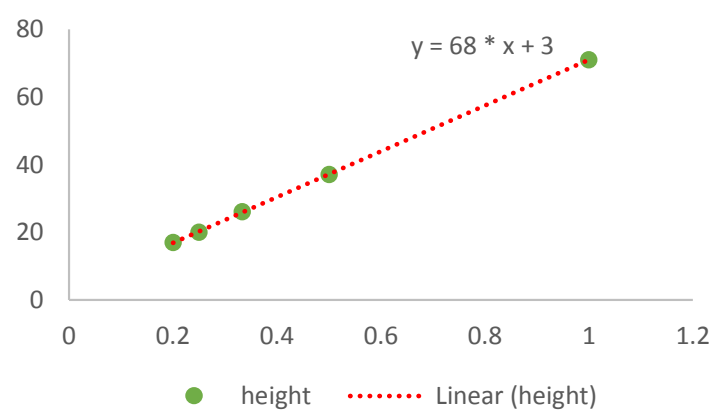

Fig. 3. Linear equation for finding the height of color region

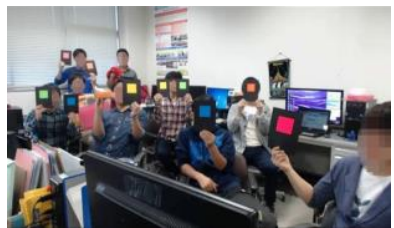

(a)

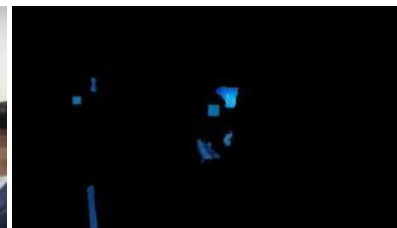

(b)
Fig. 4. (a) Input image, (b) Color mask result of size based classification of blue color

\subsection{Shape Based Classification}

The third component is the classification of card objects based on shape information. We will use the aspect ratio to classify the square color card object. The result of shape based classification is as shown in Fig. 5.

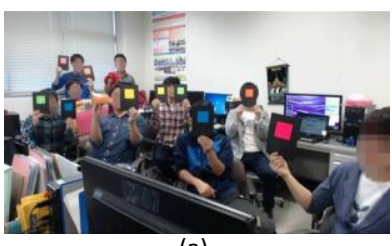

(a)

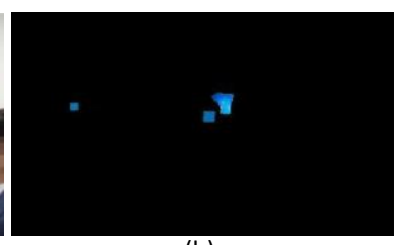

(b)
Fig. 5. (a) Input image, (b)Color mask of shape based classification of blue color.

\subsection{Black Border Detection}

The fourth component of the system is the detection of black border in order to avoid the detection of the other object that have the same color and shape as the color card objects. In Figure 6 (b), we can see that size and shape based classification result blue screen monitor as color card object because the color, size and aspect ratio of monitor is matching with the threshold value. In order to solve those kind of problem, we will use the detection of black border region around the detected color objects. We make the border region of the result of detected object by applying morphological operation. Then we remove the border region whose value is not black color. The result removing non-black border region is shown in Fig. 6.

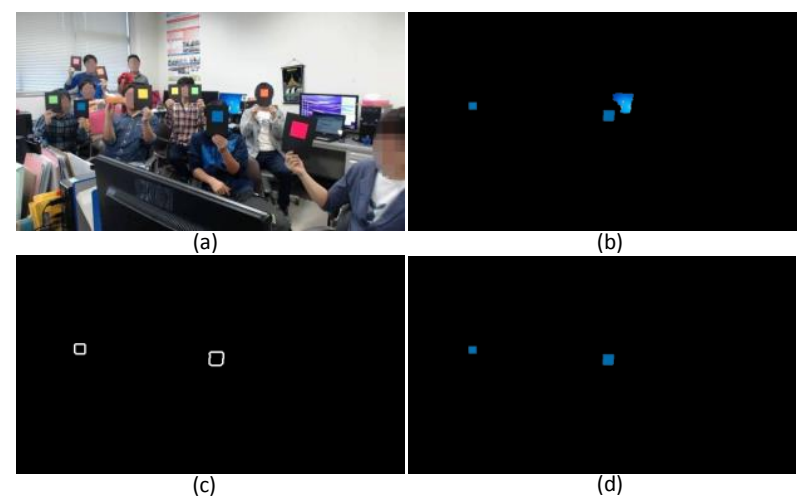

Fig. 6. (a)Input image, (b) Color mask result after size based and shape based classification for blue color, (c)Black border mask (d) Result after black border detection.

\section{Experimental Results}

We test the proposed system on the self-collecting data taking under the small classroom which includes over 10 students and large classroom which contains around 200 students at both day time and night time. In order to evaluate the robustness of the proposed methods, we also test on the images of complex environment which include the students whose dress's' color is the same as card colors. According to the experimental result, we can see that the proposed method can give accuracy of $100 \%$ for small classroom images, $93.46 \%$ for large classroom (day time) images and $88.34 \%$ for large classroom (night time) images which include highlight area and the overall accuracy is $93.93 \%$. Some experimental results of the proposed method are shown in Fig. 7. 


\section{Conclusion}

In this paper, we proposed color and shape based card images detecting and classifying method that can effectively detect and classify the various color card objects which is very useful in the implementation of the interactive e-learning system. According to experimental results, we found that the proposed methods can correctly detect and classify the card objects on both day time and night time images with accuracy over $90 \%$ and the accuracy decrease only at the highlight area of night time image due to the difficulty of color based segmentation which is the next challenge that will be solved in the future. By using the dynamic threshold calculation methods for finding the size threshold based on the lecture room size, the proposed method can give ubiquitous operation of interactive e-learning system which can process anytime and anywhere in the world.

\section{Acknowledgements}

This work was supported by JSPS KAKENHI Grant Number 15K01041.

\section{References}

1. Merten, C. and Conati, C., January. Eye-tracking to model and adapt to user meta-cognition in intelligent learning environments. In Proc. of the $11^{\text {th }}$ international conference on Intelligent user interfaces, pp. 39-46, 2006.

2. Ugurlu, Y., Human interactive e-learning systems using head posture images. In Proc. of World Academy of Science, Engineering and Technology, vol.65, pp.11071110, 2012.

3. Lee, S.H., Choi, J. and Park, J.I., Interactive e-learning system using pattern recognition and augmented reality, IEEE Transactions on Consumer Electronics, 55(2), 2009.

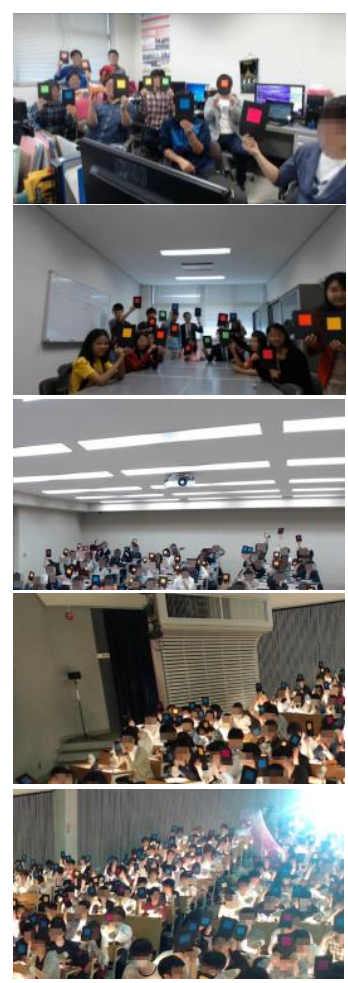

(a)

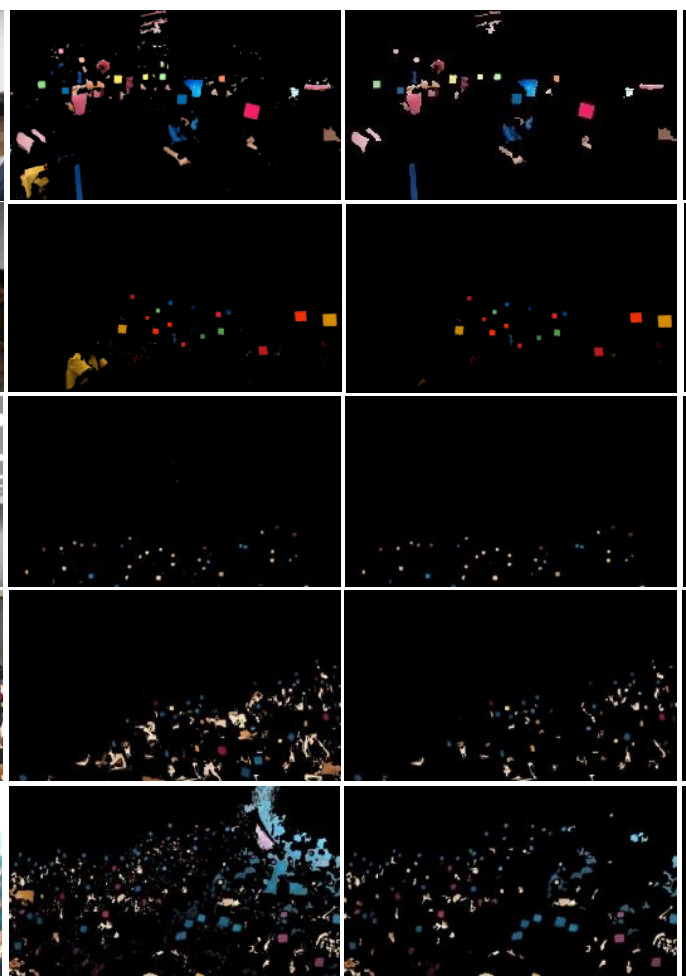

(b) (c)

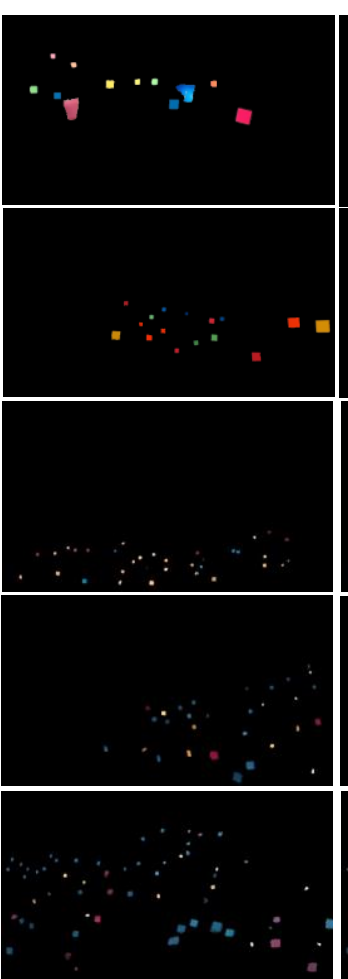

(d)

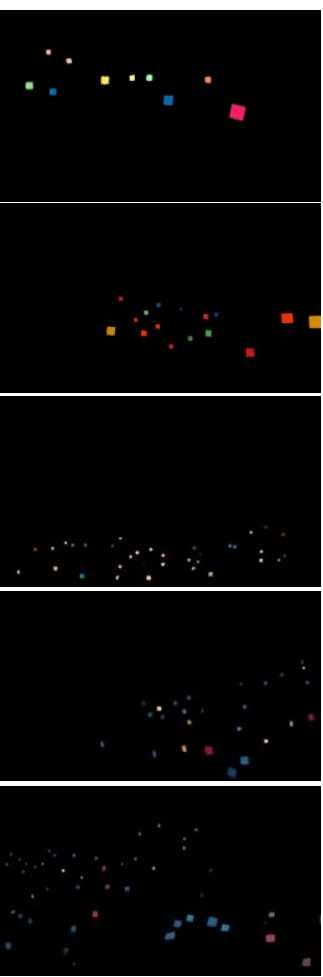

(e)

Fig. 7. Some Experimental Results [row 1 2 small class room and 3 5 large class room] (a) Input images, (b) Color segmentation result, (c) and (d) Result of size-based and shape-based classification, (e) Result of combination of color, size, shape and black border detection methods 